\title{
Avaliação do controle da asma em crianças e adolescentes do Programa Respirar da cidade de Ipatinga, Minas Gerais, Brasil
}

\author{
Evaluation of asthma control in children and adolescents in a education and \\ management program (Programa Respirar) in the city of Ipatinga, \\ Minas Gerais, Brazil
}

\author{
Bruna Almeida Lage ${ }^{1}$, Débora David De Souza ${ }^{2}$, Rosimar Freitas de Oliveira ${ }^{3}$, \\ Walquíria Carolina Catenacci Cardoso ${ }^{4}$, Lea Rache Gaspar ${ }^{5}$
}

Lage BA, Souza DD, Oliveira RF, Cardoso WCC, Gaspar LR. Avaliação do controle da asma em crianças e adolescentes do Programa Respirar da cidade de Ipatinga, Minas Gerais, Brasil / Evaluation of asthma control in children and adolescents in a education and management program (Programa Respirar) in the city of Ipatinga, Minas Gerais, Brazil. Rev Med (São Paulo). 2017 jul.-set.;96(3):165-71.

RESUMO: Introdução: A asma é uma das principais causas de morbidade na infância, ocasionando grandes prejuízos aos pacientes. O Programa Respirar foi implantado na cidade de Ipatinga, localizada na região do leste mineiro, em abril de 2003 visando ao atendimento às crianças e adolescentes asmáticos com indicação do uso de anti-inflamatório inalado profilático. $\mathrm{O}$ objetivo deste estudo foi avaliar o controle da asma dos pacientes inseridos no Programa Respirar. Métodos: estudo longitudinal retrospectivo, com 305 pacientes portadores de asma com indicação de corticoterapia inalatória, com idades entre sete e 19 anos selecionados aleatoriamente no Programa Respirar da cidade em questão, mediante análise de prontuários referentes a janeiro de 2012 a dezembro de 2015. A análise estatística foi realizada utilizando os programas EpiData (3.03) e SPSS (19.0) e para a análise descritiva, tabelas de distribuição de frequência e medidas de tendência central (média) e de variabilidade (desvio padrão, mínimo e máximo). Para a comparação das variáveis clínicas no início e após a entrada no programa, foi aplicado o teste de McNemar no caso de variáveis categóricas e teste T-pareado para as variáveis numéricas. Em todas as análises, foi considerado nível de significância de 5\%. Resultados: Quando comparadas as variáveis clínicas antes e depois de três anos de entrada no Programa Respirar, 51\% dos pacientes melhoraram a classificação da asma, $60,1 \%$ das crianças diminuíram o número de hospitalizações por crise de asma e $86,7 \%$ reduziram o número de idas ao pronto-atendimento por crises de asma. Essas diferenças foram estatisticamente significativas (valor de $\mathrm{p}<0,001$ ). Conclusão: Os pacientes assistidos pelo Programa Respirar

Apresentado na XIV Jornada Acadêmica da Saúde, V Congresso de Atualidades Médicas do Vale do Aço, Ipatinga, MG, 23 ago. 2016. 1. Acadêmica do Curso de Medicina, Instituto Metropolitano de Ensino Superior IMES - Univaço, Ipatinga, MG, BR. E-mail: brunalage_@hotmail.com.

2. Acadêmica do Curso de Medicina, Instituto Metropolitano de Ensino Superior IMES - Univaço, Ipatinga, MG, BR. e-mail: deboradavid_@hotmail.com.

3. Graduada em Ciências Biológicas, Centro Universitário de Caratinga - UNEC, Caratinga, MG, BR. Acadêmica do Curso de Medicina, Instituto Metropolitano de Ensino Superior IMES - Univaço, Ipatinga, MG, BR. E-mail: rosimar.freoli biologa@hotmail.com.

4. Graduada em Fisioterapia pela UNILESTE, Ipatinga, Minas Gerais, Brasil. Pós-graduação em Gestão em Serviço Público de Saúde pelo SENAC, Ipatinga, Minas Gerais, Brasil. Pós-graduação em Fisioterapia Hospitalar pela UNIPAC, Ipatinga, Minas Gerais, Brasil. Acadêmica do Curso de Medicina, Instituto Metropolitano de Ensino Superior IMES - Univaço, Ipatinga, MG, BR. E-mail: walquiriacatenacci@gmail.com.

5. Docente de Saúde da Criança e do Adolescente e Emergências Pediátricas do Curso de Medicina, Instituto Metropolitano de Ensino Superior IMES - Univaço, Ipatinga, MG, BR. Mestre em Ciências da Saúde pela Faculdade Federal do Rio Grande do Norte. Doutoranda no Instituto de Pesquisas Energéticas e Nucleares da USP, São Paulo, Brasil. E-mail: learache@famevaco.br.

Endereço para correspondência: Lea Rache Gaspar. Rua João Patrício, 179. Bairro Veneza I, Ipatinga, MG. CEP: 35164-251. E-mail: learache@famevaco.br. 
Lage BA, et al. Avaliação do controle da asma em crianças e adolescentes do Programa Respirar.

mantêm melhor controle sobre as crises de asma, reduzindo significativamente as hospitalizações e idas ao pronto-socorro por decorrência da doença.

Palavras-chave: Asma/prevenção \& controle; Asma/terapia; Asma/terapia; Criança; Adolescente; Planos e programas de saúde; Brasil/epidemiologia.

ABSTRACT: Introduction. Asthma is one of the main causes of childhood morbidity, causing great harm to patients. In April 2003 the Programa Respirar was implemented in the city of Ipatinga, located in the eastern region of Minas Gerais, aiming the management of asthmatic children and adolescents with a prophylactic indication to use inhaled anti-inflammatory. The purpose of this study was to evaluate the asthma control of the patients from the Programa Respirar. Methods. A longitudinal retrospective study of 305 asthma patients with indication of inhaled corticosteroid therapy, aged between seven and 19 years, randomly selected in the Programa Respirar of the city of Ipatinga, through analysis of medical records from January 2012 to December 2015. A statistical analysis was performed

\section{INTRODUÇÃO}

A asma é a doença crônica mais frequente na Linfância, constituindo um problema de saúde pública, com alto custo social e econômico no mundo ${ }^{1}$. Trata-se de uma doença inflamatória crônica das vias aéreas inferiores que causa hiperresponsividade brônquica e episódios de tosse, pressão torácica, sibilos e dispneia ${ }^{2}$.

A última revisão do consenso Global Initiative for Asthma (GINA) no ano de 2015 enfatiza o caráter heterogêneo da doença (fenótipos) na qual interagem fatores genéticos e ambientais. É uma condição que impacta a qualidade de vida e demanda do sistema público de saúde ${ }^{3,4,5}$.

Apesar do significativo avanço no tratamento e na compreensão de sua patogênese a prevalência global da doença tem aumentado nas últimas duas décadas, atingindo 6-10\% da população ${ }^{6}$. Chama a atenção que desse total, 1/3 possui idade inferior a 18 anos $^{7}$.

A população de asmáticos no Brasil é estimada em aproximadamente 20 milhões. Foram registradas 160 mil hospitalizações em 2011, abrangendo todas as faixas etárias, o que classificou a asma como a quarta causa de internações ${ }^{8}$. A doença é a segunda causa de internação hospitalar em crianças de 4 a 9 anos e a terceira em adolescentes e, nas duas faixas etárias, sendo importante causa de consultas em serviços de urgência e ambulatoriais ${ }^{9}$.

Segundo o Caderno de Atenção Primária do Ministério da Saúde ${ }^{10}$, elementos como educação sobre autocuidado e manejo adequado com medicação indicada, e orientações sobre controle ambiental são elementos fundamentais na redução do número de hospitalizações e visitas aos serviços de emergências. using the EpiData (3.03) and SPSS (19.0) programs and for the descriptive analysis, frequency distribution tables and central tendency measures (mean) and variability measures (standard deviation, minimum and maximum). For the comparison of the clinical variables at the beginning and after entry on the program, the McNemar test was applied in case of categorical variables and T-paired test for numerical variables. In all analyzes, a significance level of 5\% was considered. Results. When comparing the clinical variables before and after three years of admission to the Programa Respirar, $51 \%$ of the patients improved the classification of asthma, $60.1 \%$ of the children reduced the number of hospitalizations due to asthma attacks, and a reduction $86.7 \%$ visits to the emergency room. These differences were statistically significant ( $p$ value $<0.001$ ). Conclusion. Patients assisted by the program maintain better control over asthma attacks, significantly reducing hospital admissions and emergency room visits as a result of the disease.

Keywords: Asthma/prevention \& control; Asthma/therapy; Asthma/epidemiology; Child; Adolescent; Health programs and plans; Brazil/epidemiology.

O município de Ipatinga, com aproximadamente 239 mil habitantes, cidade de caráter industrial, grande polo siderúrgico, apresenta realidade semelhante à prevalência brasileira, com estimativa de 22.781 crianças asmáticas. Dados relativos à morbidade infantil no ano de 2000 mostram que a asma se destacava como a segunda maior causa de consultas médicas nas unidades de saúde básicas do município ${ }^{11,12}$.

A presença da asma como um fator determinante para a elevada taxa de morbidade motivou a implantação do Programa Respirar, um projeto de iniciativa municipal que visa controlar a asma em pacientes pediátricos até 19 anos que necessitam de corticoterapia inalatória com vistas à redução dos agravos da doença, responsáveis por internações e intervenções de emergência ${ }^{13}$. A sua implantação ocorreu no ano de 2003, de maneira descentralizada em todas as unidades de saúde do município ${ }^{14}$.

A implementação do programa envolveu enfermeiros, auxiliares de enfermagem, farmacêuticos, médicos, assistentes sociais; assim como demais profissionais que atuam nas unidades básicas de saúde. A capacitação dos profissionais de saúde foi realizada através da parceria entre a Secretaria Municipal de Saúde de Ipatinga e a Secretaria de Saúde do Estado de Minas Gerais, na Unidade de Referência Secundária de Pneumologia Pediátrica Campos Sales, vinculada à Secretaria Municipal de Saúde de Belo Horizonte $^{15}$.

O programa foi estruturado no fortalecimento do vínculo do paciente com a equipe da atenção primária, na capacitação e motivação dos profissionais de saúde e na assistência farmacêutica gratuita, com objetivo de possibilitar redução importante do uso de recursos de saúde e melhora da qualidade de vida desses pacientes ${ }^{14}$. 
Os três principais pilares do programa para o controle do paciente asmático são a educação associada ao tratamento farmacológico e controle ambiental.

A relação entre internações devido ao manejo inadequado da asma motivou esta pesquisa. Foi avaliado o controle da doença dos pacientes inscritos no Programa Respirar, no período de 2012 a 2015, nas unidades básicas de saúde do município de Ipatinga-MG através de análise dos seguintes indicadores de saúde: melhora dos sintomas, da classificação da asma após três anos de permanência no programa e diminuição das hospitalizações e dos atendimentos em pronto-socorro por asma.

\section{MÉTODOS}

Trata-se de um estudo longitudinal retrospectivo, baseado na análise de prontuários, realizado na cidade de Ipatinga, Minas Gerais, incluindo 8 Unidades Básicas de Saúde (UBS).

Foram selecionados aleatoriamente 305 pacientes portadores de asma com indicação de corticoterapia inalatória, com idades entre sete a 19 anos. A razão da escolha da faixa etária para esse estudo foi baseada no protocolo International Study of Asthma and Allergies in Childhood (ISAAC) ${ }^{16,17}$. Foram excluídos aqueles com prontuários com menos de $80 \%$ de preenchimento dos dados necessários para realização dos formulários da pesquisa, e crianças com menos de 7 anos.

A amostra foi calculada considerando uma população de aproximadamente 1744 inscritos no Programa Respirar e uma prevalência de redução nas internações de $36 \%{ }^{14}$, para um nível de confiança de $95 \%$ e uma margem de erro de 5\% (Open Epi versão 3.03).

Esses dados foram utilizados para preenchimento de formulários e seguinte análise das informações sobre patologias associadas, classificação da asma, número de idas ao pronto-socorro e hospitalizações no último ano antes do ingresso no programa e no ano de 2015.

Os pacientes selecionados ingressaram no Programa Respirar no ano de 2012 e foram analisadas variáveis da primeira consulta e após três anos de entrada, na última consulta de 2015. O acompanhamento médico era feito mensalmente no início do uso da medicação, e após o paciente atingir o controle, trimestralmente. $\mathrm{O}$ paciente recebe alta ambulatorial após um ano mantendo o controle da doença. Em todas as consultas eram preenchidos, o número de exacerbações, idas ao pronto-socorro, hospitalizações, classificação da asma, necessidade de uso de medicação de alívio e patologias associadas, entre outros $^{15}$.

Os indivíduos receberam gratuitamente toda medicação inalatória de alívio e para controle da doença em longo prazo, espaçadores valvulados e medicamento para controle de rinite alérgica, que é a principal comorbidade associada $^{18}$. O controle da adesão medicamentosa foi realizado pelo farmacêutico de cada unidade de saúde. $\mathrm{O}$ programa Respirar adotava um modelo desenvolvido para o controle do tempo de uso da medicação, onde se anota a data de entrega do medicamento e do término do mesmo. No momento da entrega do medicamento o farmacêutico confirmava pelo frasco utilizado se todas as doses foram utilizadas $^{15}$.

Em todas as consultas foi abordado um conteúdo educacional programático feito de forma verbal e escrita para o paciente, incluindo os fatores desencadeantes e agravantes da asma e orientação de como evitá-los, avaliação de técnica inalatória, além de descrição da diferença entre medicação controladora e de resgate, conhecimento sobre os efeitos colaterais dos medicamentos usados e informações de como minimizá-los e elucidação de temores ou mitos. Todo paciente participou de uma palestra educativa por semestre, até um ano após o controle. Neste período o paciente recebia novamente orientações educacionais, medicamentos, inclusive os imunobiológicos especiais como as vacinas contra influenza e pneumococo ${ }^{15}$.

O critério eleito para diagnóstico e classificação da asma como controlada, parcialmente controlada e não controlada foi baseado nas diretrizes do GINA. Foi considerado controle da asma quando o paciente apresentava melhora da classificação da doença, bem como a redução em atendimentos no pronto-socorro e do número de hospitalizações ${ }^{4}$.

Os pacientes foram classificados na primeira consulta quando inseridos no programa e em todo retorno era feita nova classificação de acordo com seu quadro clínico atual, sendo essa classificação realizada pelo médico da UBS. Os pacientes não foram classificados pela gravidade porque optamos utilizar a classificação de acordo com o controle. A classificação considerando a gravidade é feita de acordo com a dose de medicação utilizada, o que não foi o foco desta pesquisa.

As análises estatísticas foram realizadas usando os programas EpiData (3.03) e SPSS (19.0). A análise descritiva foi realizada por meio de tabelas de distribuição de frequência e medidas de tendência central (média) e de variabilidade (desvio padrão, mínimo e máximo). Para a comparação das variáveis clínicas no início e após a entrada no programa, foram utilizados os testes de McNemar para variáveis categóricas e teste T-pareado para as variáveis numéricas. Em todas as análises, foi considerado nível de significância de 5\%.

Esta pesquisa foi aprovada pelo Comitê de Ética em Pesquisa da Unileste/MG, cujo Protocolo de Pesquisa é $\mathrm{n}^{\circ}$ 58.312.12 e Ofício 17/12. 
Lage BA, et al. Avaliação do controle da asma em crianças e adolescentes do Programa Respirar.

\section{RESULTADOS}

Os aspectos demográficos da pesquisa demonstraram que, dos 305 participantes, $113(37,05 \%)$ eram do sexo feminino e 192 (62,95\%) do sexo masculino. A média de renda familiar dos pacientes foi de $2,2( \pm 1,3)$ salários.

A média de idade do início dos sintomas foi de 14 ( \pm 20) meses. Dentre as comorbidades alérgicas associadas, a rinite foi a patologia mais comum, $220(73,6 \%)$ pacientes, seguida da dermatite atópica em $105(35,1 \%)$ crianças.

Cento e noventa $(190,65,5 \%)$ indivíduos possuíam história familiar de asma, 172 (66,2\%) história de atopia familiar e 88 (33,8\%) negaram histórico familiar alérgico.

Na comparação das variáveis clínicas antes e depois de três anos de entrada no Programa Respirar, observouse que $151(51 \%)$ pacientes melhoraram a classificação da asma; 173 (60,1\%) crianças diminuíram o número de hospitalizações por crise; e 247 (86,7\%) dos participantes reduziram o número de idas ao pronto-atendimento por crises asmáticas. Essas diferenças foram estatisticamente significativas (valor de $\mathrm{p}<0,001$ ).

Não houve diferença estatisticamente significativa no controle da asma em pacientes de diferentes unidades básicas de saúde.
Tabela 1. Características dos participantes $(\mathrm{N}=\mathbf{3 0 5})$

\begin{tabular}{l|r}
\hline Gênero & \\
\hline Masculino & $192(62,95 \%)$ \\
\hline Feminino & $113(37,05 \%)$ \\
\hline Renda familiar (salários) & $2,2( \pm 1,3)$ \\
\hline Idade de início dos sintomas (meses) & $14( \pm 20)$ \\
\hline Rinite & \\
\hline Sim & $220(73,6 \%)$ \\
\hline Não & $75(26,4 \%)$ \\
\hline Dermatite atópica & $105(35,1 \%)$ \\
\hline Sim & $194(64,9 \%)$ \\
\hline Não & \\
\hline História de atopia familiar & $172(66,2 \%)$ \\
\hline Sim & $88(33,8 \%)$ \\
\hline Não & \\
\hline História familiar de asma & $190(65,5 \%)$ \\
\hline Sim & $100(34,5 \%)$ \\
\hline Não & \\
\hline
\end{tabular}

Tabela 2. Comparação de variáveis clínicas antes e após 3 anos de entrada no programa

\begin{tabular}{l|c|c|c}
\hline & Frequência & Porcentagem válida & Valor de P \\
\hline Número de hospitalizaçães & & & \\
\hline Diminuição & 173 & $56,7 \%$ & $<0,001^{* *}$ \\
\hline Sem redução & 115 & $37,7 \%$ & \\
\hline Total & 288 & & \\
\hline Número de idas ao PS & & & \\
\hline Diminuição & 247 & $86,7 \%$ & $<0,001^{* *}$ \\
\hline Sem redução & 38 & $13,3 \%$ & \\
\hline Total & 285 & & \\
\hline Classificação da asma & & & \\
\hline Melhora da classificação & 151 & $51,0 \%$ & \\
\hline Sem melhora da classificação & 145 & $49,0 \%$ & \\
\hline Total & 296 & & \\
\hline
\end{tabular}

*McNemar **T-pareado

\section{DISCUSSÃO}

A asma é uma condição clínica e o objetivo principal de sua abordagem é alcançar o controle da doença ${ }^{19}$. Nos últimos 40 anos observou-se um aumento significativo da doença e importante relação entre o agravamento da enfermidade e a duração da doença. O estudo de Angnes et al. ${ }^{20}$, constatou que, ao longo dos anos, há uma limitação física, emocional e social do paciente e piora da intensidade das crises asmáticas, aumentando o risco de evolução com desfecho fatal.
Apesar de todos os avanços realizados nas últimas décadas no tratamento e implementação de diretrizes para melhora do manejo da doença, o controle da asma ainda é um desafio ${ }^{21,22}$. Entre as possíveis explicações para esse fato, estão a prescrição médica inadequada ou a utilização da medicação prescrita de forma incorreta ${ }^{21}$.

As iniciativas de implantação de programas de controle da asma no Brasil possibilitaram resultados animadores. Esses programas têm como objetivo disponibilizar medicamentos, promover abordagem integral do paciente e manejo preventivo em conformidade com a 
Lage BA, et al. Avaliação do controle da asma em crianças e adolescentes do Programa Respirar.

\section{Global Initiative for Asthma $a^{4}$.}

Os dados do Sistema Único de Saúde (SUS) mostram a redução do número de hospitalizações por asma, de $400 \mathrm{mil} / \mathrm{ano}$ para menos de $200 \mathrm{mil} /$ ano entre os anos de 2000 e 2012, e redução de $30 \%$ do gasto bruto, por hospitalizações com asmáticos (R $\$ 110$ milhões vs. R \$ 80 milhões). Portanto é possível supor que os programas de asma implantados nos municípios brasileiros ao longo dos últimos anos auxiliaram nessa redução de internações ${ }^{18}$.

Os dados obtidos nesta pesquisa mostram que a promoção da educação em asma pelas ações do Programa Respirar trouxe benefícios significativos para população asmática e suas famílias. É importante associar o processo educativo ao atendimento de rotina a todos os pacientes asmáticos. O presente estudo mostrou que uma parcela significativa de crianças participantes do Programa Respirar conseguiu controlar melhor seu quadro de asma reduzindo significativamente o número de hospitalizações, assim como o índice de idas ao pronto-socorro.

O percentual de controle da asma foi menos significativo que o de redução de hospitalizações e idas ao pronto-socorro, pois alguns pacientes não se sentiam doentes, com isso não faziam uso adequado da medicação e o controle da asma não era alcançado. Pelo mesmo motivo, não iam aos serviços de urgência, reduzindo significativamente a utilização do mesmo. Além disso, antes de entrarem no programa os pacientes não tinham educação em asma, procurando os serviços de urgência e emergência, às vezes, sem necessidade. Após receberem todas as informações sobre a doença, a grande maioria conseguia identificar os sinais e sintomas de crise e précrise e faziam uso da medicação de forma adequada em seu domicílio, reduzindo a frequência de hospitalizações e idas ao pronto-socorro.

Esta pesquisa foi concordante com o estudo de Fontes et al. ${ }^{23}$, que mostrou redução no número de hospitalizações por asma e atendimentos em serviço de urgência, de 895 para $180(79,9 \%)$ e de 5.375 para 713 $(86,8 \%)$, respectivamente após implantação do programa de atendimento a pacientes asmáticos em Belo Horizonte.

Semelhantes resultados também foram observados em outras cidades como Feira de Santana e Salvador, onde foi constatada redução de $42 \%$ e $90 \%$, respectivamente, nas hospitalizações por asma, indicando um impacto significativo na morbidade por asma e consequentemente nos gastos do Sistema Único de Saúde com a doença ${ }^{24,25}$.

Stirbulov et al. ${ }^{26}$ descreveram a respeito das diretrizes de manejo da asma, enfatizando que a meta principal para o manejo é o controle. Salienta que a educação em saúde, aliada à terapia medicamentosa, é a base do tratamento e prevenção da asma, proporcionando qualidade de vida.

A capacitação contínua de todas as categorias de profissionais da saúde em todos os níveis da atenção se faz necessária, assim como do paciente e seus familiares ${ }^{27}$.
Quando o paciente tem conhecimento sobre manejo, controle e cuidados com a asma há melhora na profilaxia das crises, proporcionando redução dos atendimentos no serviço de emergência. É importante a presença de profissionais capacitados para fornecer orientações sobre o conceito de asma, reconhecimento dos fatores agravantes, identificação das crises e ações para seu controle, utilização correta da medicação, dos dispositivos inalatórios, conhecimentos básicos sobre as medicações e importância da terapia. Uma intervenção educativa aliada ao tratamento clínico é fundamental e imprescindível no controle da asma ${ }^{20}$.

A adesão medicamentosa é parte importante do tratamento e a classe de medicamentos mais efetiva para o tratamento da asma persistente consiste nos corticosteroides inalatórios. Um aspecto importante no estudo realizado por Dalcin et al..$^{28}$ foi a abordagem educativa, focalizando a necessidade de adesão a longo prazo ao tratamento com essa medicação, resultando em seu maior uso.

O impacto clínico mais tardio relacionado ao uso dos corticosteroides inalatórios sobre os sintomas de asma, quando comparado com o alívio imediato que as drogas broncodilatadoras inalatórias proporcionam, parece ser um ponto importante para a não adesão à sua prescrição, o que pode justificar a persistência, após o processo educativo, de um grupo de pacientes que não aderem a essa medicação. As drogas $\beta 2$-agonistas inalatórias de longa ação, quando associadas aos corticosteroides inalatórios, compõem a estratégia mais efetiva para controlar a asma moderada e grave ${ }^{4}$.

Em seu estudo, Rifaat et al. ${ }^{29}$ concluíram que, entre os diversos fatores que podem modular a adesão ao tratamento em pacientes asmáticos, a educação em asma é o fator ouro, sendo o mais importante em aumentar a adesão à terapia com corticosteroides inalatórios. Quando a não adesão é identificada, o sucesso pode não ser alcançado. Além disso, é importante lembrar que as definições de asma grave e as orientações para o manejo de fenótipos específicos são todas baseadas na suposição de que o paciente está aderindo ao tratamento ${ }^{30}$. Entre os temores mais comuns que impedem o uso normal dos costicosteroides inalatórios, está o risco potencial de dependência (60\%) e preocupação com os efeitos colaterais dos esteroides (41\%), como retardo do crescimento ${ }^{29}$.

Vieira et al. ${ }^{31}$ concordam que é necessário um acompanhamento mais detalhado a respeito das condições de habitação do asmático, dos hábitos de vida e do provimento de informações sobre a asma para o manejo adequado dessa doença. $\mathrm{O}$ controle da asma pode ser feito por meio das ações de educação aos pacientes e sua família e pela utilização adequada dos medicamentos ${ }^{32}$.

Há, portanto, uma necessidade de que fontes de financiamento permaneçam suficientemente sólidas, garantindo acesso gratuito a medicamentos e acompanhamento sistemático de pacientes asmáticos pela 
Lage BA, et al. Avaliação do controle da asma em crianças e adolescentes do Programa Respirar.

atenção primária ${ }^{33}$.

A limitação deste estudo é referente à percepção ruim dos pacientes sobre os seus sintomas, pois às vezes eles relatam melhora que não se evidencia no exame físico, isso pode interferir na adesão medicamentosa, pois se sentindo bem eles não utilizam adequadamente a medicação e isso dificulta o controle dos pacientes.

\section{CONCLUSÃO}

O Programa Respirar é um programa de atendimento a asmáticos destinado à população pediátrica, estruturado no fortalecimento do vínculo do paciente com a unidade básica de saúde (UBS), e equipe de profissionais da saúde capacitada e motivada, aliada à assistência farmacêutica gratuita.

\section{Quadro. Formulário de Pesquisa}

A1. Número do formulário:

A2. UBS:

A3. Nome:

A4. Prontuário/sanitas:

A5. Data de nascimento:

\section{I- Variáveis demográficas e socioeconômicas: \\ B1. Gênero: Masculino ( ) Feminino ( ) \\ B2. Renda familiar (salários):}

\section{II- Variáveis clínicas e ambientais na primeira consulta do Programa Respirar:}

C1. Idade de início dos sintomas (meses):

C2. História familiar de asma: Sim ( ) Não ( )

C3. Atopia familiar: Sim ( ) Não ( )

C4. Classificação da asma: 1. Controlada ( ); 2. Parcialmente controlada ( ); 3. Não controlada ( ).

C5. Doenças associadas:

C5.1. Rinite: 1. Sim ( ); 2. Não ( )

C5.2. Dermatite atópica: 1. Sim ( ); 2. Não ( )

C5.3. Outros:

C6. Número de hospitalizações por crise de asma nos últimos

12 meses antes de entrar no programa:

C7. Número de idas ao pronto atendimento por crise de asma

nos últimos 12 meses antes de entrar no programa: ....

\section{III-Variáveis clínicas e ambientais após entrada no programa respirar:}

D1. Classificação da asma: 1. Controlada ( ); 2. Parcialmente controlada ( ); 3. Não controlada ( ).

D2. Número de hospitalizações por crise de asma nos últimos 12 meses:

D3. Número de idas ao pronto atendimento por crise de asma nos últimos 12 meses:
Neste estudo mostramos que os pacientes assistidos pelo Programa Respirar, no período de 2012 a 2015, mantiveram melhor controle sobre as crises de asma, permanecendo assintomáticos por maior período de tempo.

Houve ainda uma redução significativa no número de hospitalizações e de idas ao pronto-socorro para atendimentos de urgência por asma nos pacientes asmáticos inseridos no programa Respirar. O programa Respirar apresenta, portanto, um impacto positivo sobre o controle da asma.

Esta pesquisa mostrou como esse programa baseado em educação individualizada possui a enorme capacidade de interferir positivamente no quadro das crianças e adolescentes asmáticos de Ipatinga. Estes resultados são animadores e podem ser replicados em municípios com características semelhantes com alta prevalência da doença.

\section{REFERÊNCIAS}

1. Coriolano MWL, Lima MM, Queiroga BAM, Moreno LR, Lima LS. Educação permanente com agentes comunitários de saúde: uma proposta de cuidado com crianças asmáticas. Trab Educ Saude (Rio de Janeiro). 2012;10(1):37-59. doi: http:// dx.doi.org/10.1590/S1981-77462012000100003.

2. Smeltzer SC, Bare BG, Hinkle JL, Cheever KH. Tratado de enfermagem médico-cirúrgico. 11a ed. Rio de Janeiro: Guanabara Koogan; 2009.

3. Pinto LA, Stein RT, Kabesch M. O impacto da genética na asma infantil. J Pediatr (Rio J.) 2008;84(4):S68-S75. Doi: http://dx.doi.org/10.1590/S0021-75572008000500010.

4. Global Initiative for Asthma (GINA). Global strategy for asthma management and prevention: NHLBI/WHO Workshop Report. Bethesda: National Institute of Health; 2015.

5. Souza AMV, March MFP. Política sanitária para asma no Brasil. Rev Pediatria SOPERJ. 2010;11(1):10-18. Disponível em: http://revistadepediatriasoperj.org.br/detalhe_artigo. asp?id $=568$.

6. Lugogo NL, Kraft M. Epidemiology of asthma. Clin Chest Med. 2006;27(1):1-15. doi: http://dx.doi.org/10.1016/j. ccm.2005.10.006.

7. Global Initiative for Asthma (GINA). Global strategy for asthma management and prevention 2006. NHLBI/WHO Workshop Report. Bethesda: National Institute of Health; 2006. Available from: http://www.who.int/respiratory/asthma/ GINA_WR_2006_copyright[1].pdf.

8. Sociedade Brasileira de Pneumologia e Tisiologia. Diretrizes da Sociedade Brasileira de Pneumologia e Tisiologia para o Manejo da Asma - 2012. J Bras Pneumol. 2012;38(Supl 1):S1S46. Disponível em: http://www.jornaldepneumologia.com.br/ pdf/suple_200_70_38_completo_versao_corrigida_04-09-12. pdf.

9. Asher MI, Montefort S, Björkstén B, Lai CKW, Strachan DP, Weiland SK, Willians H, ISAAC Phase Three Study Group. Worldwide time trends in the prevalence of symptoms of asthma, allergic rhinoconjunctivitis, and eczema in childhood: ISAAC Phases One and Three repeat multicountry crosssectional surveys. Lancet. 2006;26;368(9537):733-43. doi: 10.1016/S0140-6736 (06) 69283-0. 
Lage BA, et al. Avaliação do controle da asma em crianças e adolescentes do Programa Respirar.

10. Brasil. Ministério da Saúde. Caderno de Atenção Básica. Doenças respiratórias crônicas. Brasília; 2010 [citado 9 mar. 2016]. Disponível em: http://bvsms.saude.gov.br/bvs/ publicacoes/doencas_respiratorias_cronicas.pdf.

11. Brasil. Ministério da Saúde. DATASUS. Sistema de Informações Hospitalares do SUS (SIH/SUS). Brasília; 2008 [citado 4 fev. 2016]. Disponível em: http://datasus.saude.gov. $\mathrm{br} /$ sistemas-e-aplicativos/hospitalares/sihsus.

12. Secretaria Municipal de Saúde de Ipatinga. Dados estatísticos. Morbidade 2000 [28 jan 2016]. Disponível em: www.ipatinga mg.gov.br. html.

13. Gaspar LR, Gaspar GR, Campos Júnior FM, Campos MF. Impacto de um programa de asma do SUS, em IpatingaMG, na qualidade de vida da criança e do adolescente com indicação de corticoterapia inalatória. In: $13^{\circ}$ Congresso Brasileiro de Pneumologia Pediátrica; Salvador, BA, 09-11 out. 2011.

14. Programa Cidades Sustentáveis. Programa Respirar Ipatinga/MG apud DATASUS. Brasília; 2013 [citado 4 fev. 2016]. Disponível em: http://indicadores.cidadessustentaveis. org.br/br/MG/ipatinga/boa-pratica/100/programa-respirar.

15. Gaspar LR, Lima LGCS. Projeto de estruturação municipal da assistência pública a asma - Programa Respirar/IpatingaMG. 2015.

16. Fontes MJF, Fonseca MTM, Camargos PAM, Affonso AGA, Calazans GMC. Asma em menores de cinco anos: dificuldades no diagnóstico e na prescrição da corticoterapia inalatória. J Bras Pneumol. 2005;31(3):244-53. doi: http://dx.doi. org/10.1590/S1806-37132005000300011.

17. Solé D, Camelo-Nunes IC, Wandalsen GF, Mallozi MC. A asma na criança e no adolescente brasileiro: contribuição do International Study of Asthma and Allergies in Childhood (ISAAC). Rev Paul Pediatr. 2014;32(1):114-25. doi: http:// dx.doi.org/10.1590/S0103-05822014000100018.

18. Brasil. Ministério da Saúde. DATASUS. Informações epidemiológicas de morbidade (TABNET) e mortalidade. Brasília; 2014 [citado 7 abr. 2016]. Disponível em: http:// www2.datasus.gov.br/DATASUS.

19. Schatz M, Zeiger RS, Vollmer WM, Mosen D, Cozinheiro EF. Determinants of future long-term asthma control. J Allergy Clin Immunol. 2006;118(5):1048-53. doi: http://dx.doi. org/10.1016/j.jaci.2006.07.057.

20. Angnes MR, Macagnan JBA, Cauduro JM, Silveira L. Asma: uma revisão da literatura. Rev Saude Publ Santa Cat (Florianópolis). 2012;5(3):81-94. Disponível em: http://esp. saude.sc.gov.br/sistemas/revista/index.php/inicio/article/ viewFile/146/196.

21. Barnes PJ. The size of the problem of managing asthma. Respir Med. 2004;98(Suppl B):4-8. doi: https://doi. org/10.1016/j.rmed.2004.07.009.

22. Dalcin PTR, Menegotto DM, Zanonato A, Franciscatto L, Soliman F, Figueiredo M, Pereira RP. Factors associated with uncontrolled asthma in Porto Alegre, Brazil. Braz J Med Biol Res. 2009;42(11):1097-103. doi: http://dx.doi.org/10.1590/ S0100-879X2009005000035.

23. Fontes MJF, Affonso AGA, Calazans GMC, Andrade CR, Lasmar LMLBF, Nader CMFF, Camargos PAM. Impacto de um programa de manejo da asma sobre as hospitalizações e os atendimentos de urgência. J Pediatr (Rio J.). 2011;87(5):4128. doi: http://dx.doi.org/10.2223/JPED.2129.

24. Brandão H, Silva Junior I, Neves Neto J, Amaral D, Cruz C, Souza-Machado A, Cruz AA. Impacto do programa para o controle da asma e da rinite (proar) de Feira de Santana, Bahia. Gaz Med Bahia. 2008;78(2):64-8. Disponível em: http://www.gmbahia.ufba.br/ojs/index.php/gmbahia/article/ viewFile/751/734.

25. Ponte E, Franco RA, Souza-Machado A, Souza-Machado C, Cruz AA. Impacto de um programa para o controle da asma grave na utilização de recursos do Sistema Único de Saúde. J Bras Pneumol. 2007;33(1):15-9. doi: http://dx.doi. org/10.1590/S1806-37132007000100006.

26. Stirbulov R, Bernd LAG, Sole D. IV Diretrizes brasileiras para o manejo da Asma. J Bras Pneumol. 2006;32(7):447-74. doi: http://dx.doi.org/10.1590/S1806-37132006001100002.

27. Stelmach R, Cerci Neto A, Fonseca ACCF, Ponte EV, Alves G, Araújo-Costa IN, et al. Programas e centros de atenção a asmáticos no Brasil; uma oficina de trabalho: revisitando e explicitando conceitos. J Bras Pneumol. 2015;41(1):3-15. doi: http://dx.doi.org/10.1590/S1806-37132015000100002.

28. Dalcin PTR, Grutcki DM, Laporte PP, Lima PB, Viana VP, Konzen GL, Menegotto SM, Fonseca MA, Pereira RP. Impacto de uma intervenção educacional de curta duração sobre a adesão ao tratamento e controle da asma. J Bras Pneumol. 2011;17(1):19-27. doi: http://dx.doi.org/10.1590/ S1806-37132011000100005.

29. Rifaat N, Abdel-Hady E, Hasan AA. The golden factor in adherence to inhaled corticosteroid in asthma patients. Egyptian J Chest Dis Tuberculosis. 2013;62:371-6. doi: https://doi.org/10.1016/j.ejcdt.2013.07.010.

30. Lindsay JT, Heaney LG. Nonadherence in difficult asthma facts, myths, and a time to act. Dove Press Med J. 2013;7:32936. doi:10.2147 / PPA.S38208.

31. Vieira JWC, Silva AA, Oliveira FM. Conhecimento e impacto sobre o manejo das crises de pacientes portadores de Asma. Rev Bras Enfermagem. 2008;61(6):853-7. doi: http://dx.doi. org/10.1590/S0034-71672008000600010.

32. Carmo TA, Andrade SM, Cerci Neto A. Avaliação de um programa de controle da asma em unidades de saúde da família. Cad Saúde Pública. 2011;27(1):162-72. doi: http:// dx.doi.org/10.1590/S0102-311X2011000100017.

33. Amaral LM, Palma PV, Leite ICG. Considerações sobre a asma de interesse para a atenção primária: epidemiologia, impacto econômico e políticas públicas. Rev APS. 2012;15(4):508-16. Disponível em: https://aps.ufjf.emnuvens.com.br/aps/article/ view/1897/683.

Participação dos autores no artigo: Lage BA. Colaboração no artigo: Preenchimento de formulário e auxílio na elaboração da discussão; Souza DD. Colaboração no artigo: Preenchimento de formulário, elaboração de métodos e resultados, auxílio na elaboração da introdução, auxílio nas correções sugeridas pela revista para o artigo; Oliveira RF. Colaboração no artigo: Preenchimento de formulário, elaboração da discussão e conclusão, correções sugeridas pela revista para o artigo e check list final; Cardoso WCC. Colaboração no artigo: Preenchimento de formulário e auxílio na elaboração da introdução; Gaspar LR. Colaboração no artigo: Coordenação e coleta de dados. 